\title{
Smiles and frowns in migration/velocity analysis
}

\author{
Jinming Zhu', Laurence R. Lines ${ }^{2}$ and Samuel H. Gray ${ }^{3}$ \\ ${ }^{1}$ GX Technology Corp., 5847 San Felipe, Suite 3500, Houston, TX77057 \\ 2 Dept. of Geology and Geophysics, University of Calgary, Calgary, Alberta, Canada T2N 1N4 \\ 3 Amoco Canada Petroleum Co., P. O. Box 200, Station M, Calgary, Alberta, Canada T2P 2H8
}

\section{INTRODUCTION}

Depth migration can be defined as the process of positioning reflected seismic arrivals at their proper subsurface locations. The accurate depth migration of seismic reflectors requires accurate velocity estimations. Inaccurate velocity estimates will cause moveout artifacts such as "smiles" and "frowns" to appear on depth migrated images. The elimination of these moveout features by the adjustment of seismic velocities allows depth migration to be used as a powerful velocity analysis tool. It can be argued on the basis of model studies (Lines et al., 1993) and with real data examples (Whitmore and Garing, 1993) that iterative prestack depth migration provides a very general velocity analysis method for structurally complex media.In this paper, we examine migration moveout for both poststack and prestack depth migration of point diffractors both mathematically and geometrically. Point diffractors are used due to their simplicity and the fact that reflected wavefields can be considered as a superposition of point diffractor arrivals according to Huygens' principle. We show the effects of velocity on the depth migration of point diffraction arrivals so that one can establish criteria for velocity analysis. We do this for both zero offset (poststack) and non-zero offset (prestack) recording configurations.

\section{SMILES AND FROWNS IN POSTSTACK MIGRATION}

In understanding the case of poststack migration, consider two point diffractors in the middle of a uniform medium with velocity $v=4,000 \mathrm{~m} / \mathrm{s}$. The depths of the diffractors are $600 \mathrm{~m}$ and $800 \mathrm{~m}$ respectively. We now examine the migration of a point diffraction seismogram from this model recorded by coincident source-receiver positions. The zero-offset section is shown in Figure 1 . The images obtained by migrating the input diffraction hyperbola in Figure 1 are compared for the cases where the velocity is too slow (Figure 2a), exactly correct (Figure 2b) and the case where the velocity is too high (Figure 2c). If

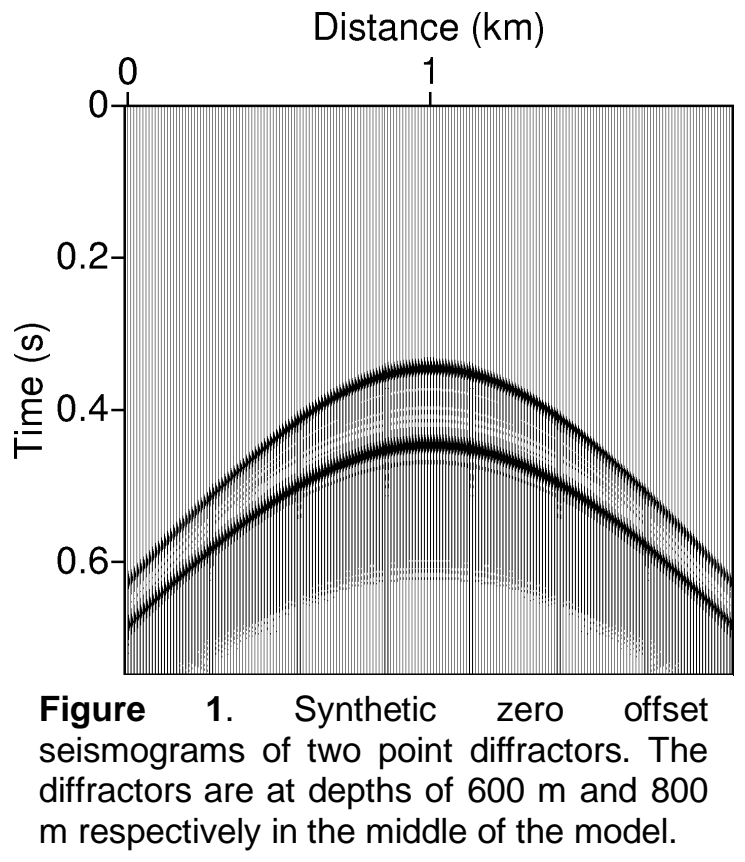



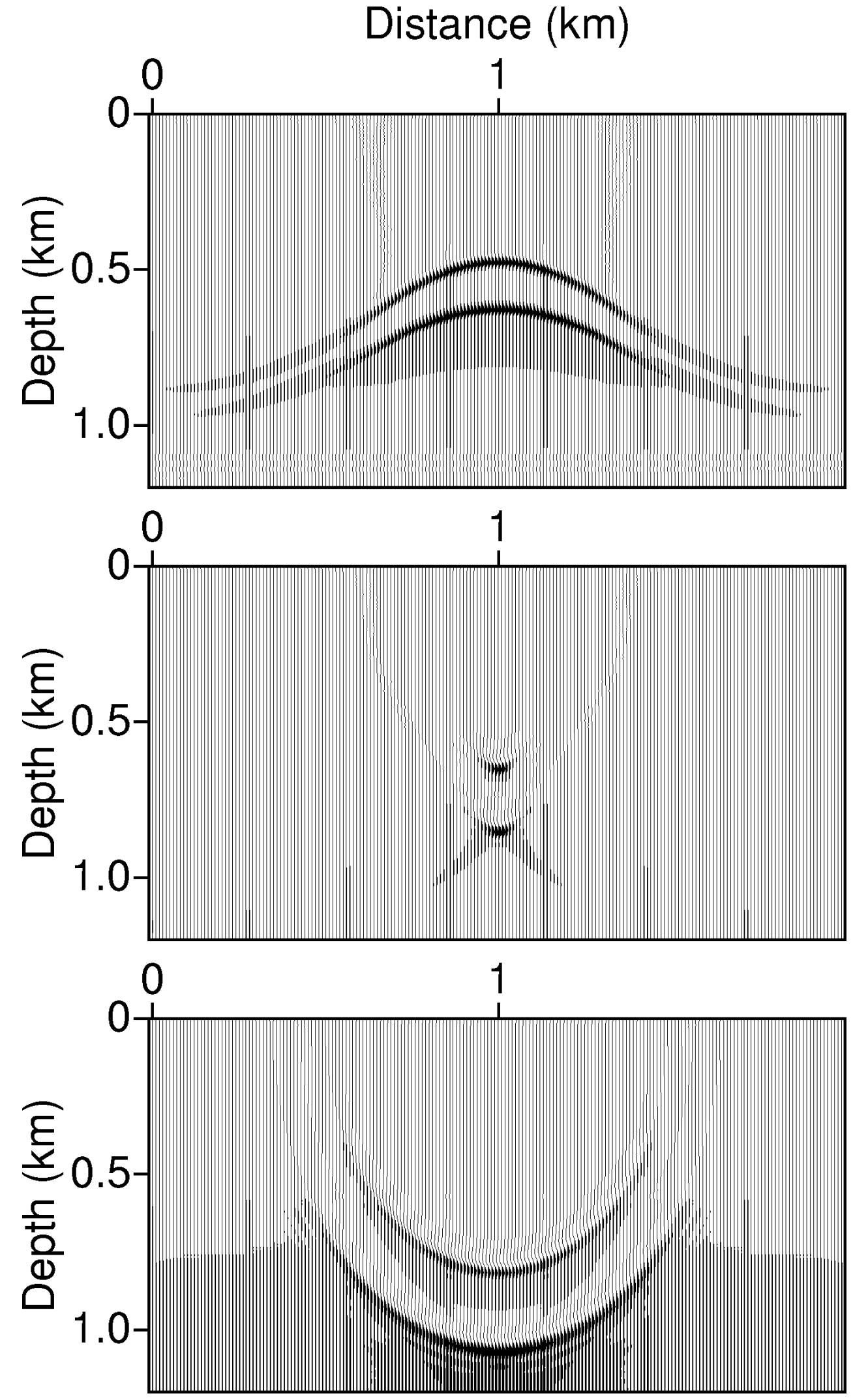

Figure 2. Poststack migration of a two point diffractors model. (a) A smaller migration velocity produces shallow "frowns", (b) The true velocity collapses the hyperbolae to concentrated blobs and (c) a larger migration velocity results in deep "smiles". 
the velocity is too low, the poststack migration images are shallow "frowns". If the velocity is correct, the images are concentrated blobs at the proper depth. If the velocity is high, the images are deep "smiles". These have been described by data examples in Yilmaz (1987). The shallow "frowns" are described as

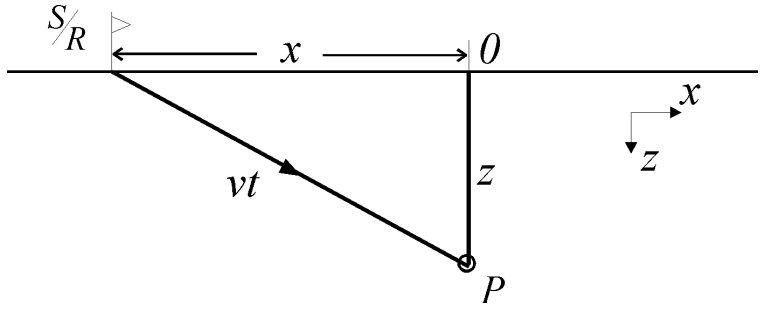

Figure 3. The zero offset (poststack) geometry for a point diffractor at $P$.

"undermigration" while the deeper smiles are described as "overmigration". The phenomena of undermigrations and overmigrations for poststack data can be described by considering the case of coincident source-receiver positions. For the purpose of description simplicity, we consider the case of a point diffractor for a coincident source-receiver (or zero offset) recording configuration. This recording configuration, as shown in Figure 3 is the situation which we simulate with stacked data.

Suppose there is a point diffractor $\mathrm{P}$ on the vertical axis of a Cartesian system $(x, z)$. The diffractor at depth $z$ is vertically below the origin. Consider a coincident source-receiver pair $\mathrm{S} / \mathrm{R}$ on the surface of the earth with lateral offset $x$ from the origin. Let the one-way vertical traveltime from the surface to $\mathrm{P}$ be $t_{0}$; let the total traveltime from $\mathrm{S} / \mathrm{R}$ to $\mathrm{P}$ be $t$; and let the medium velocity be $v$. The one-way traveltime for an arrival traveling from $\mathrm{S} / \mathrm{R}$ to $\mathrm{P}$ is given by using the Pythagorean theorem and the distance relationship between the sides of the right angle triangle in Figure 3. That is

$$
x^{2}+v^{2} t_{0}^{2}=v^{2} t^{2}
$$

or,

$$
t=\sqrt{t_{0}^{2}+\frac{x^{2}}{v^{2}}}
$$

If we wish to use the two-way reflection times $\left(2 t\right.$ and $\left.2 t_{0}\right)$ which are the arrival times for a reflection experiment, we can consider the same distance relationship by using the medium's half velocity, $v / 2$. Then the model in Figure 3 is essentially the "exploding reflector" model of Loewenthal et al. (1976). In this model, reflection seismograms containing arrivals with two-way propagation are described by one-way propagation of "explosions" which propagate to the surface with half the velocity of the medium. Except for a few pathological cases (Claerbout, 1985; Yilmaz, 1987),

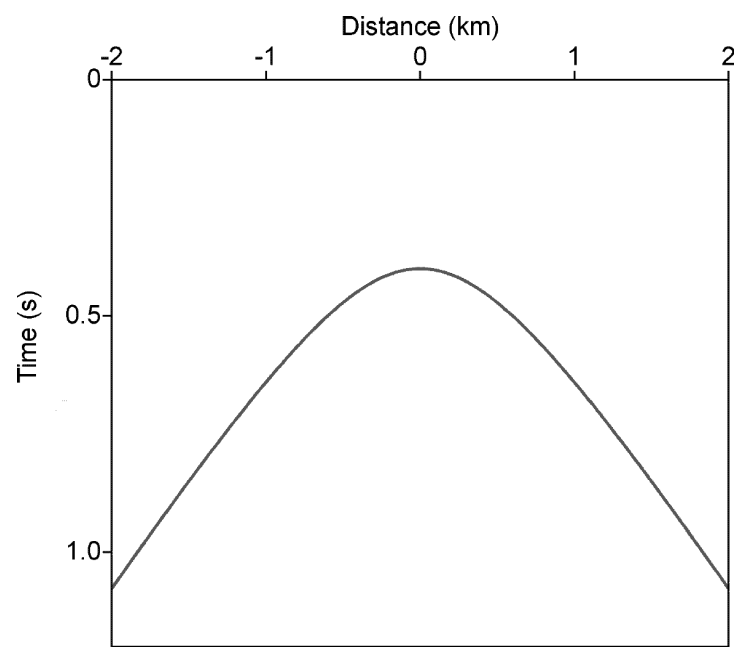

Figure 4. Geometrical expression of a zero offset section for a point diffractor. 
this "exploding reflector" model can be considered as a suitable model for poststack data. Figure 4 is a record of such an "explosion" experiment from the diffractor model.

Let us consider the migration of a diffraction arrival of a trace at offset $x$ from the origin. Suppose the arrival time is $t$. To migrate this arrival, we use the principal of aplanatic surfaces (Sheriff, 1991). An aplanatic surface defines the locus of possible reflection depth points that could exist for a given one-way traveltime, $t$, and a migration velocity, $v_{m}$. For a coincident source-receiver position and a constant migration velocity, $v_{m}$, the aplanatic surface is defined by the following equation of a circle giving all possible locations of reflection points,

$$
\left(x-x_{m}\right)^{2}+z_{m}^{2}=v_{m}^{2} t^{2}
$$

where $\left(x_{m}, z_{m}\right)$ defines the migrated domain. If we substitute the expression for $t$ from equation (2), we obtain

$$
z_{m}^{2}=v_{m}^{2}\left(t_{0}^{2}+\frac{x^{2}}{v^{2}}\right)-\left(x_{m}-x\right)^{2}
$$

Note that the observed reflection time in (2) is expressed in terms of the actual velocity, $v$, which we generally do not know but hope to determine, whereas the estimated velocity used in migration is $v_{m}$. Migration of the record in Figure 4 is essentially the superposition of all these aplanatic surfaces one for each sourcereceiver pair. We hope to find geometric criteria corresponding to cases where $v_{m}<v, v_{m}=v$ and $v_{m}>v$ which will allow us to find cases where the velocity is correct.

In migration we are dealing with the superposition of wavefield amplitudes that are distributed along aplanatic surfaces. For the poststack situation, this is the "wavefront superposition method" described by Robinson and Treitel (1980). The migrated image represents a summation of those amplitudes that are in phase such that they will constructively interfere. Mathematically, this constructive interference can be described by the method of stationary phase (Scales, 1995). The basic idea of stationary phase is that highly oscillatory time sequences tend to cancel upon migration except where the phase function has a stationary point. This stationary point occurs where the first derivative of the phase function equals zero. In migration, the phase function is the phase difference between the migration curve and the diffraction signatures of the data; locations where the migration curve is tangent to diffraction or reflection events in the data give the stationary phase contributions to the migration. This stationary point occurs where the first derivative of the phase function equals zero.

In migration, an alternative kinematic description of the amplitude summation along aplanatic surfaces is given by the envelope curves for the aplanatic surfaces. Is a set of aplanatic curves is described by $F(x, z, t)=0$, then its envelope is defined by curves satisfying $F(x, z, t)=0$ and $d F / d x=0$. For doing this, we need to find the 
envelope of the aplanatic curves defined by (4), as shown by Maeland (1989). Essentially the envelope is the solution of the system consisting of equation (4) and its tangent curve (Sneddon, 1957). The tangent curve of (4) is given by differentiating equation (4) with respect to the source-receiver position $x$,

$$
\frac{v_{m}^{2}}{v^{2}} x-\left(x-x_{m}\right)=0
$$

or equivalently if we define $\beta=v_{m} / v$,

$$
\left(1-\beta^{2}\right) x=x_{m}
$$

Now let's consider the case of $v_{m} \neq v$. For this case, we can have,

$$
x=\frac{x_{m}}{1-\beta^{2}}
$$

Substituting (7) into (4) leads to,

$$
\frac{z_{m}^{2}}{v_{m}^{2} t_{0}^{2}}-\frac{x_{m}^{2}}{\left(v^{2}-v_{m}^{2}\right) t_{0}^{2}}=1
$$

Now let's consider three specific cases.

\section{Migration velocity smaller than the medium velocity, i.e., $v_{m}<v$}

In this case, equation (8) represents a hyperbola. Its vertex is on the depth axis with coordinate of $v_{m} t_{0}$ below the origin. The apex is thus above the diffractor position, as $v_{m} t_{0}<v t_{0}$. Obviously, it opens downward because the center of the hyperbola is just on the coordinate origin. Thus, the poststack migration of the diffraction curve will be a shallow hyperbolic frown when the migration velocity is too small, so that undermigration partially collapses the original hyperbola into a second, better focused hyperbola. This observation forms the basis of residual migration and cascaded migration.

In fact, the formation of such shallow frowns can also be well illustrated geometrically. Figure 5 illustrates this migration case. The light grey circle is the diffractor point. The dark grey curve is the original record we simulated for such an "explosion". The near black curves are the migration aplanatics which finally superpose to form the envelope of another hyperbola (in light grey) but is laterally much narrowed. This essentially indicates undermigration.

\section{Migration velocity greater than the medium velocity, i.e., $v_{m}>v$}

In this case, equation (8) can effectively be reformulated as, 


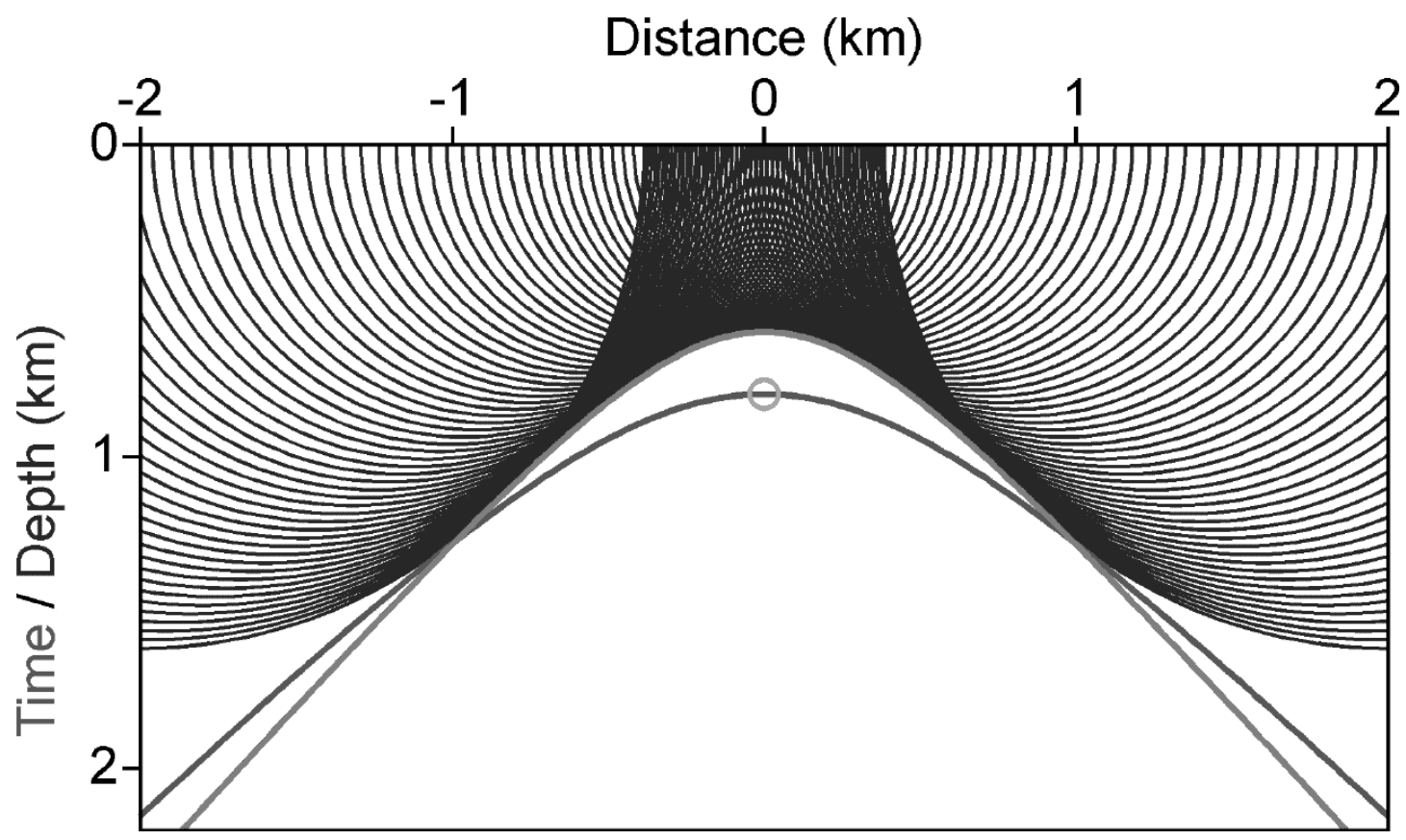

Figure 5. Poststack migration of the diffractor model when a smaller $(3 \mathrm{~km} / \mathrm{s})$ than the true velocity $(4 \mathrm{~km} / \mathrm{s})$ is used. The light grey circle represents the diffractor position. The dark grey is the scaled recorded hyperbolic diffraction curve. The superposition of all the wavefronts in near black results in a shrunken hyperbola in light grey in the final migrated section.

$$
\frac{z_{m}^{2}}{v_{m}^{2} t_{0}^{2}}+\frac{x_{m}^{2}}{\left(v_{m}^{2}-v^{2}\right) t_{0}^{2}}=1
$$

This is the equation of a semi-ellipse with the center at the coordinate origin. The vertex on the depth $z$ axis is still $z_{0} t_{0}\left(>v t_{0}\right)$, which is now above the diffractor point P. The mouth of the ellipse is toward the negative axis of depth as we are only interested in the positive $z$ values. Therefore, the migration of the diffraction curve in Figure 4 will be deep elliptic smiles on the migrated section when the migration velocity is too large.

Such a migration procedure is also geometrically represented in Figure 6. Most of the curves in Figure 6 are just the same as in Figure 5. Figure 6 excellently shows that when the migration velocity is too high, the superposition of the individual aplanatics forms an elliptic smile (in green) in the migrated section.

\section{Migration velocity equal to the medium velocity, i.e., $v_{m}=v$}

In this case, we have to start from equations (4) and (6), as equation (8) is no longer valid. When $v_{m}=v$, (6) gives $x_{m}=0$. Substituting this value of $x_{m}$ into (4), we obtain $z_{m}=v t_{0}=z$. This implies that when the migration velocity is correct the superposition of the migration aplanatics finally collapse the recorded diffractions to its correct spatial position, $\left(x_{m}, z_{m}\right)=(0, z)$. Parallel to such mathematical 


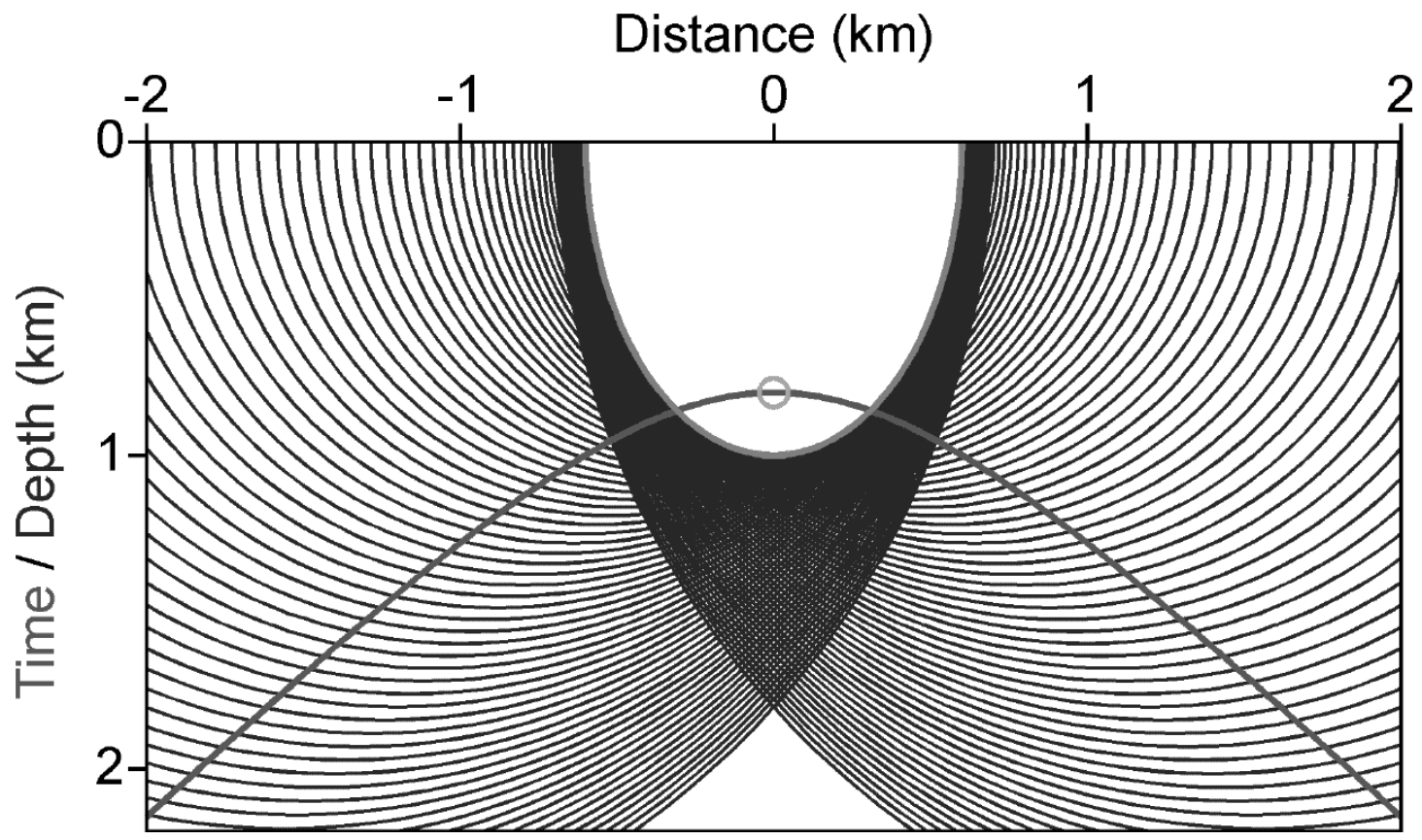

Figure 6. Poststack migration of the diffractor model when a larger $(5 \mathrm{~km} / \mathrm{s})$ than the true velocity $(4 \mathrm{~km} / \mathrm{s})$ is used. The superposition of all the wavefronts in near black results in an elliptic smile in light grey in the final migration section.

development, Figure 7 geometrically expresses the procedure of reconstructing the true diffraction point by simple superposition of aplanatic curves.

\section{SMILES AND FROWNS IN PRESTACK MIGRATION SECTION}

In this section, we will show that the smiles and frowns discussed above are also common to prestack migrated stacked sections. They can also be easily verified both mathematically and geometrically. As the mathematical development is very similar to that in the poststack migration case, we will thus focus on the geometrical aspects.

Let's first consider the same point diffractor $\mathrm{P}$ on the vertical axis of a Cartesian system. Now assume there are a source at $\mathrm{S}$ and a receiver at $\mathrm{R}$ on the earth's surface, with x coordinate of $x_{s}$ and $x_{r}$ respectively (see Figure 8 ). If the medium velocity is $v$, the total traveltime from the source $\mathrm{S}$ to the diffractor $\mathrm{P}$, and back up to the receiver $\mathrm{R}$, can thus be given by the so-called double square root relationship (Claerbout, 1985),

$$
t=\frac{1}{v}\left(\sqrt{x_{s}^{2}+z^{2}}+\sqrt{x_{r}^{2}+z^{2}}\right)
$$

Migration of a single diffraction arrival at $\mathrm{R}$ due to the source at $\mathrm{S}$ can still be described by the concept of aplanatic surfaces. The aplanatic surface for the sourcereceiver pair as shown in Figure 8, analogous to equation (3) can be represented by,

$$
\sqrt{\left(x_{s}-x_{m}\right)^{2}+z_{m}^{2}}+\sqrt{\left(x_{r}-x_{m}\right)^{2}+z_{m}^{2}}=v_{m} t
$$




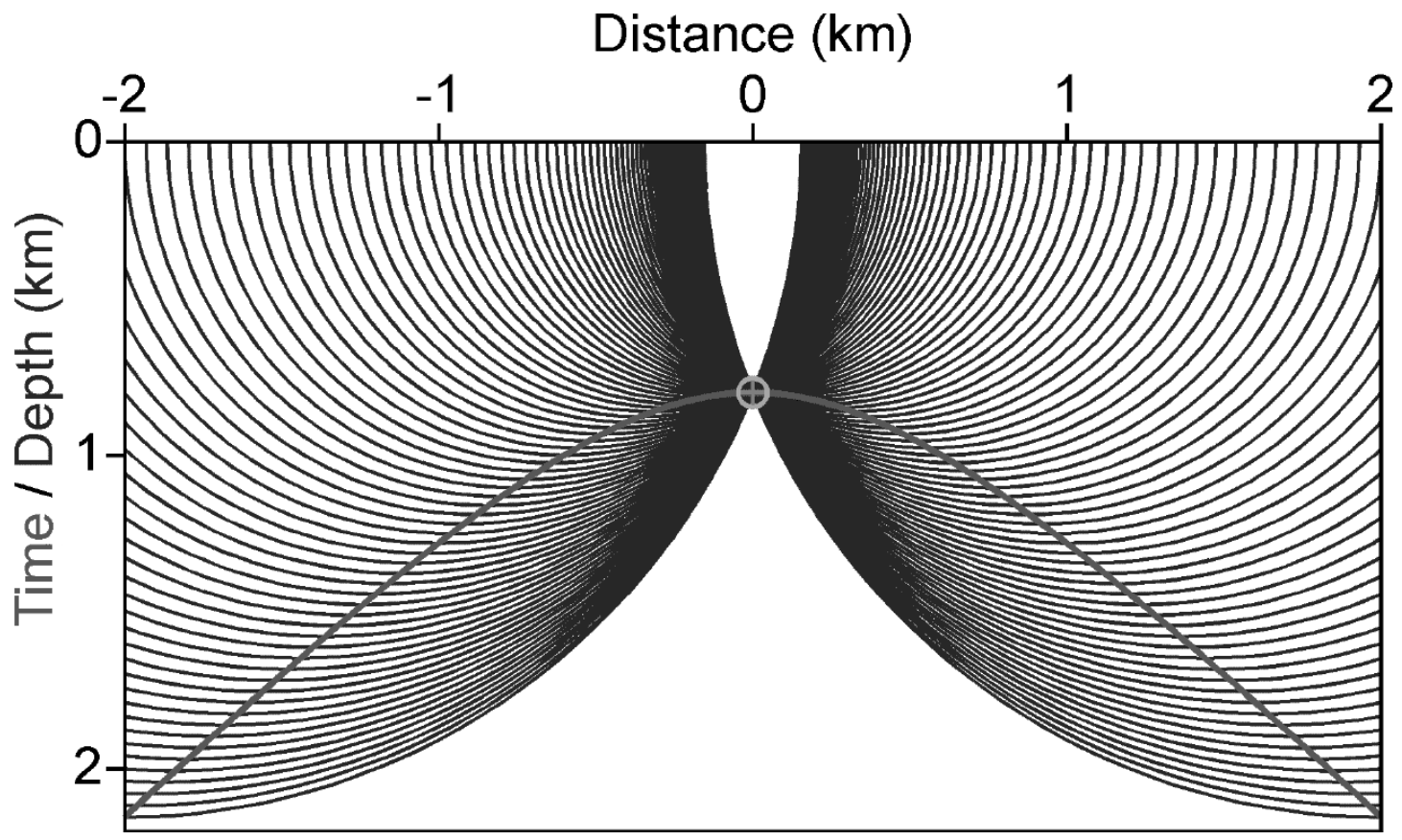

Figure 7. Poststack migration of the diffractor model when the true velocity $(4 \mathrm{~km} / \mathrm{s})$ is used for migration. The dark grey cross is the result of the constructive superposition of all the wavefronts in near black. This indicates a perfect recovery of the diffractor point in the migration.

Substituting (10) into the above, we have,

$$
\begin{aligned}
F\left(x_{m}, z_{m} ; x_{s}, x_{r}\right) & =\beta\left\lfloor\sqrt{x_{s}^{2}+z^{2}}+\sqrt{x_{r}^{2}+z^{2}}\right\rfloor \\
& -\left[\sqrt{\left(x_{s}-x_{m}\right)^{2}+z_{m}^{2}}+\sqrt{\left(x_{s}-x_{m}\right)^{2}+z_{m}^{2}}\right]=0
\end{aligned}
$$

This is essentially an ellipse in the migrated space $\left(x_{m}, z_{m}\right)$ for this special case of constant velocity.

The final migration of all these arrivals recorded by different receivers due to many sources is the envelope of these individual ellipses. The envelope of these ellipses is essentially the solution of equation (12) and its derivative equations (Sneddon, 1957),

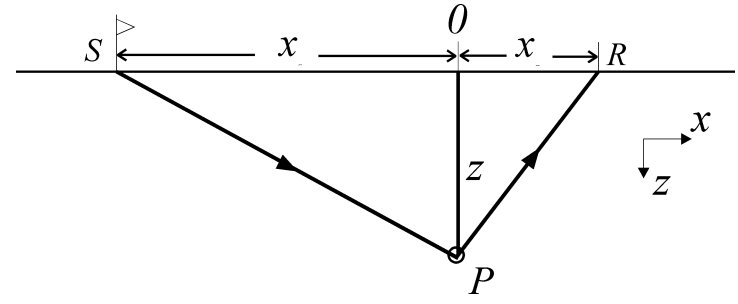

Figure 8. Prestack geometry for a point diffractor at $P$.

$$
\begin{aligned}
& F_{x_{s}}\left(x_{m}, z_{m} ; x_{s}, x_{r}\right)=0 \\
& F_{x_{r}}\left(x_{m}, z_{m} ; x_{s}, x_{r}\right)=0
\end{aligned}
$$


Following the same procedures as in the last section, we can develop the same conclusions as those in the poststack migration, though the mathematical derivations will be much more complicated. Figure 9 schematically shows four shot gathers from the diffraction model as shown in Figure 8. Figure 10 geometrically summarizes the migration procedure when too small a velocity is used for prestack migration. Just as expected, the superposition of all individual migration ellipses results in a shrunken hyperbola (in light grey). Notice, however, that not all the ellipses are tangent to the envelope. Figure 12 illustrates that the correct velocity

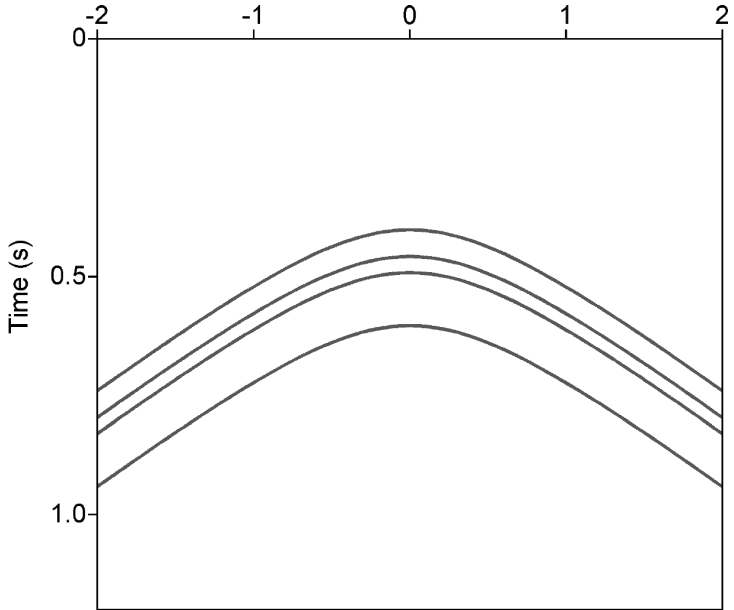

Figure 9. Some representative prestack recording events from a diffractor point model. Each event corresponds to a source position. The diffractor lies at 0.8 $\mathrm{km}$ depth in the middle of the model.

allows the migration to reconstruct the point diffractor model almost perfectly as long as the recording coverage is sufficiently wide and dense. In contrast to Figure 10, Figure 11 demonstrates that when too large a velocity is used for prestack migration $\left(v_{m}>v\right)$, the superposition of individual elliptical aplanatics (in near black) results in another ellipse (in light grey) in the final migrated section.

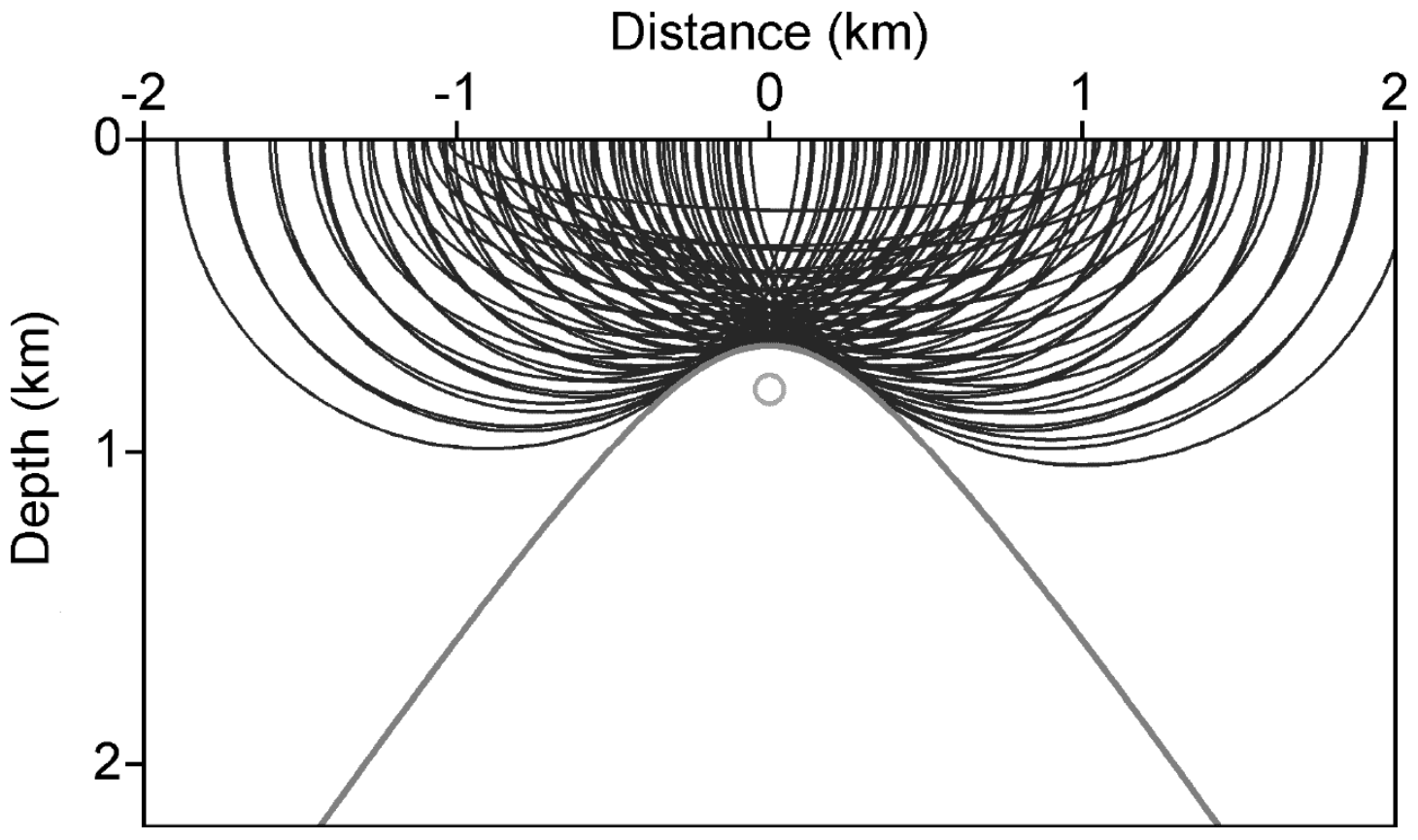

Figure 10. Prestack migration of the diffractor model when a smaller $(3.3 \mathrm{~km} / \mathrm{s})$ than the true velocity $(4 \mathrm{~km} / \mathrm{s})$ is used. The light grey circle represents the diffractor position. The superposition of all the wavefronts in near black results in a shrunken hyperbola in light grey in the final migration section. 


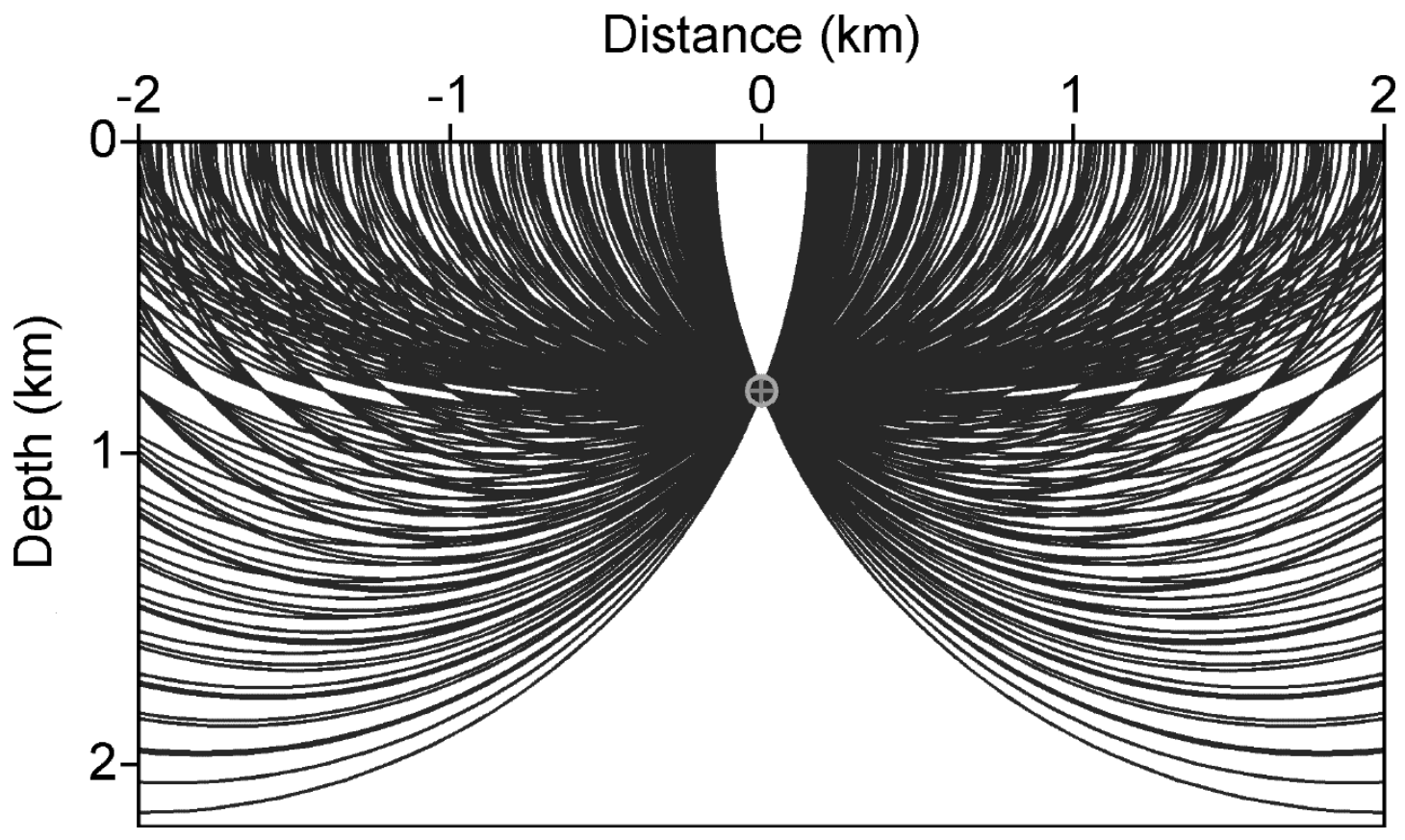

Figure 11. Prestack migration of the diffractor model when the true velocity $(4 \mathrm{~km} / \mathrm{s})$ is used for migration. The dark grey cross is the result of the constructive superposition of all the wavefronts in near black. This indicates a perfect recovery of the diffractor point in the migration.

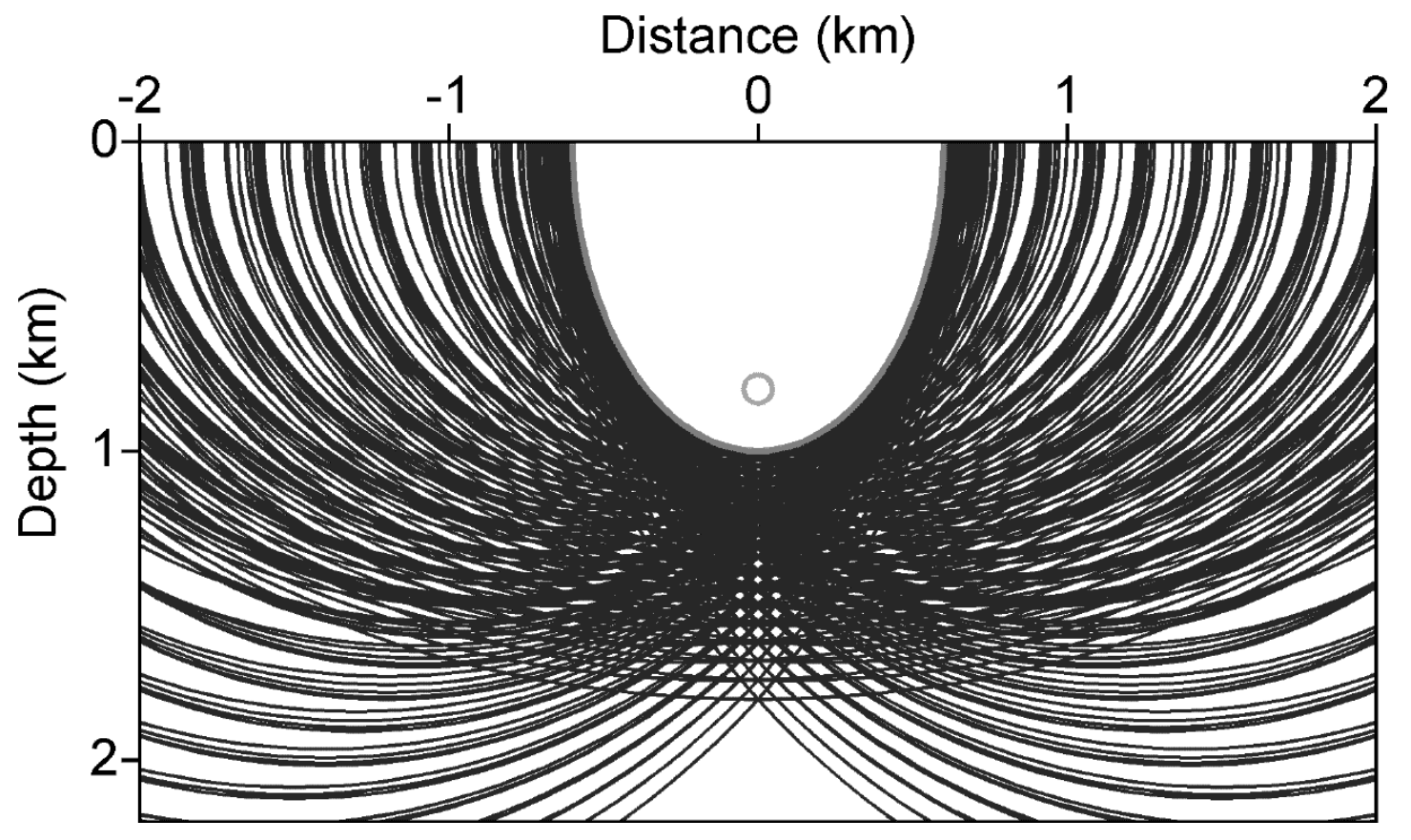

Figure 12. Prestack migration of the diffractor model when a larger $(5 \mathrm{~km} / \mathrm{s})$ than the true velocity $(4 \mathrm{~km} / \mathrm{s})$ is used for migration. The superposition of all the wavefronts in near black results in an elliptic smile in light grey in the final migration section. 


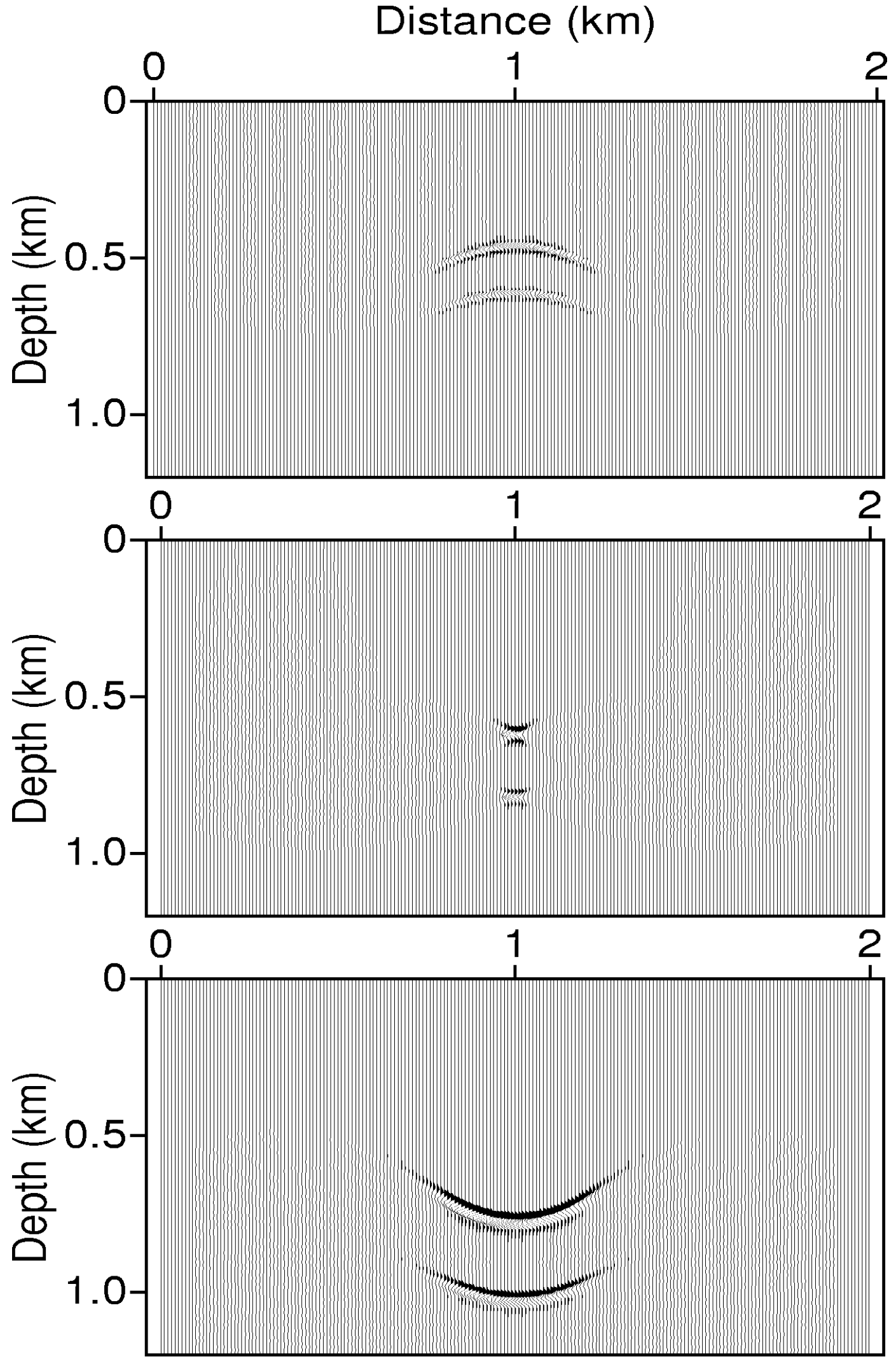

Figure 13. Prestack depth migration of a two point difractors model. A migration velocity smaller than, equal to nd larger than the true velocity is used for the top, middle and bottom images respectively. 
Figure 13 shows a numerical example of prestack depth migrations which correspond to cases of velocity lower than, equal to, and higher than the true medium velocity. A low migration velocity $\left({ }^{v_{m}}=3,000 \mathrm{~m} / \mathrm{s}\right)$ results in frowning migratedimage (top), caused by an insufficient collapse of diffractions. In contrast, using a velocity that is too high $\left(v_{m}=5000 \mathrm{~m} / \mathrm{s}\right)$ in migration results in smiling images (bottom). In either of the above two cases, the migrated images of the diffractors are not properly focused. A smaller velocity results in image shallowing while a larger velocity leads to image deepening. Only when the true velocity is used, will the diffractions completely collapse to their true positions (center). Thus, the final migrated section exhibits "smiles" and "frowns" patterns whether the migration is performed on poststack or prestack seismic data whenever errors exist in the migration velocity.

These undermigration and overmigration features can thus be generally observed in both poststack and prestack migration sections. However, these smiles and frowns are generally very difficult to observe on the migration sections as individual diffractors are often just an element of the reflecting interface. It is generally the migration moveout effects on common image gathers (CIGs) from prestack migrations that are quite pronounced and allow for effective velocity analysis. Interestingly enough, the moveout characteristics on common image gathers (CIGs) are very different from those in the migrated stacked sections.

\section{PRESTACK DEPTH MIGRATION MOVEOUT}

Many recent studies demonstrate that prestack depth migration can accurately image reflections and diffractions without dip restriction if a reasonable approximation of the velocity field is available (Versteeg, 1991; Lines et al., 1993). Nevertheless, the velocity model is the key component in these migrations. Theoretically, there exist several alternative methods for velocity analysis (Versteeg, 1993; Lines et al., 1993). Here we will analyze the prestack migration moveout features which are fundamental to the basic theories in interval velocity analysis utilizing CIGs (Al-Yahya, 1989). Our analysis, however, will no longer depend on the assumption of a layered earth model.

Consider the general subsurface structure and recording geometry as shown in Figure 14. $\mathrm{P}$ denotes the arbitrary scattering point in the earth's interior. $\mathrm{S}$ and $\mathrm{R}$ are a source-receiver pair illuminating P. D is the surface image of P. Assuming that the averaging velocity above $\mathrm{P}$ is $\bar{v}$, and that the diffraction received at $\mathrm{R}$ due to a source wavelet from $\mathrm{S}$ and then diffracted at $\mathrm{P}$ never travels beneath $\mathrm{P}$, then its arrival time can be expressed as:

$$
t=\frac{1}{v}(\overline{S P}+\overline{R P})
$$

When an incorrect average velocity $\bar{v}_{m}$ is used for migration, the diffraction signal received from $\mathrm{P}$ will be migrated to an incorrect point $\mathrm{P}^{\prime}$. $\mathrm{P}^{\prime}$ generally has both 


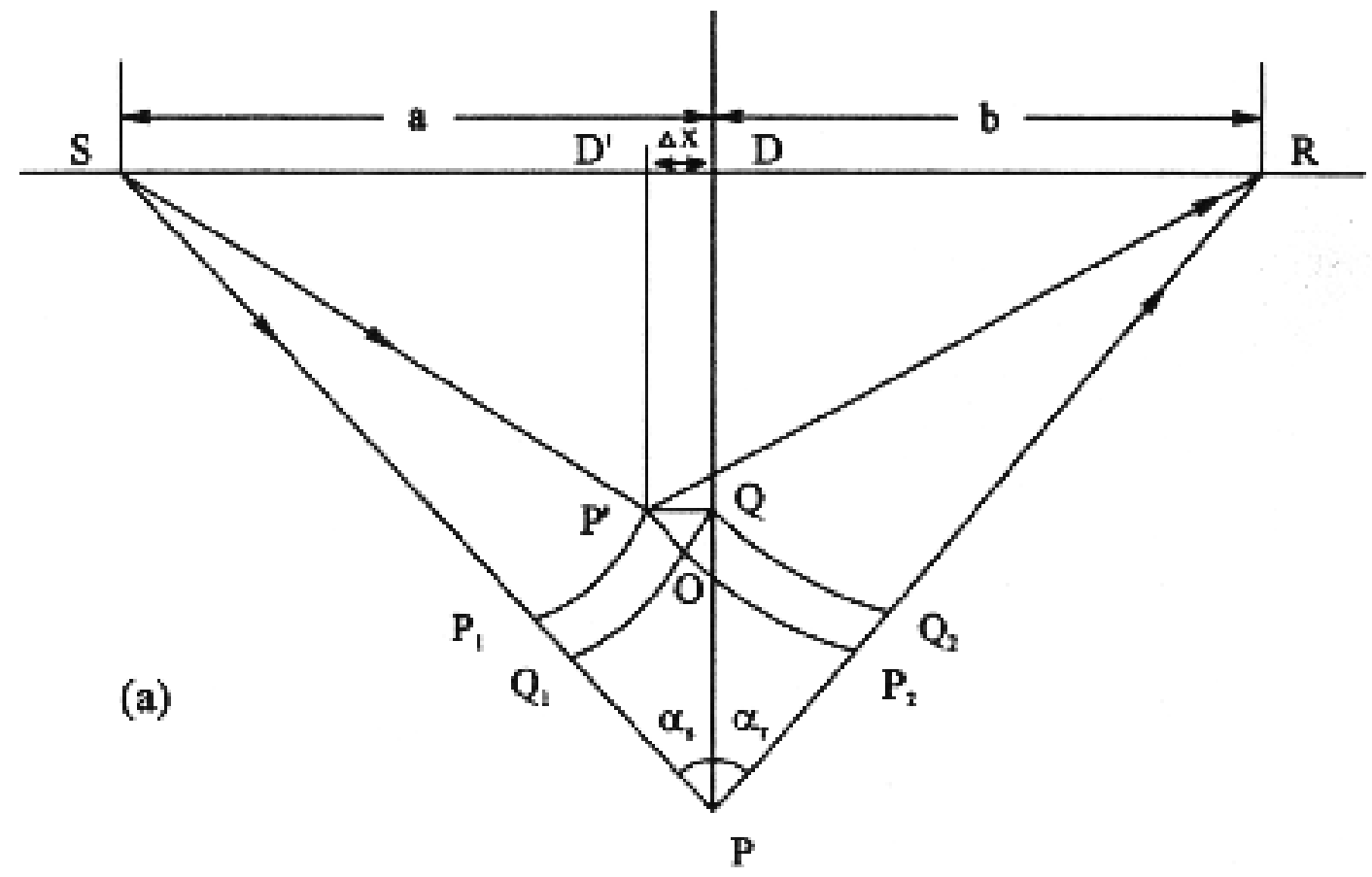

(b)

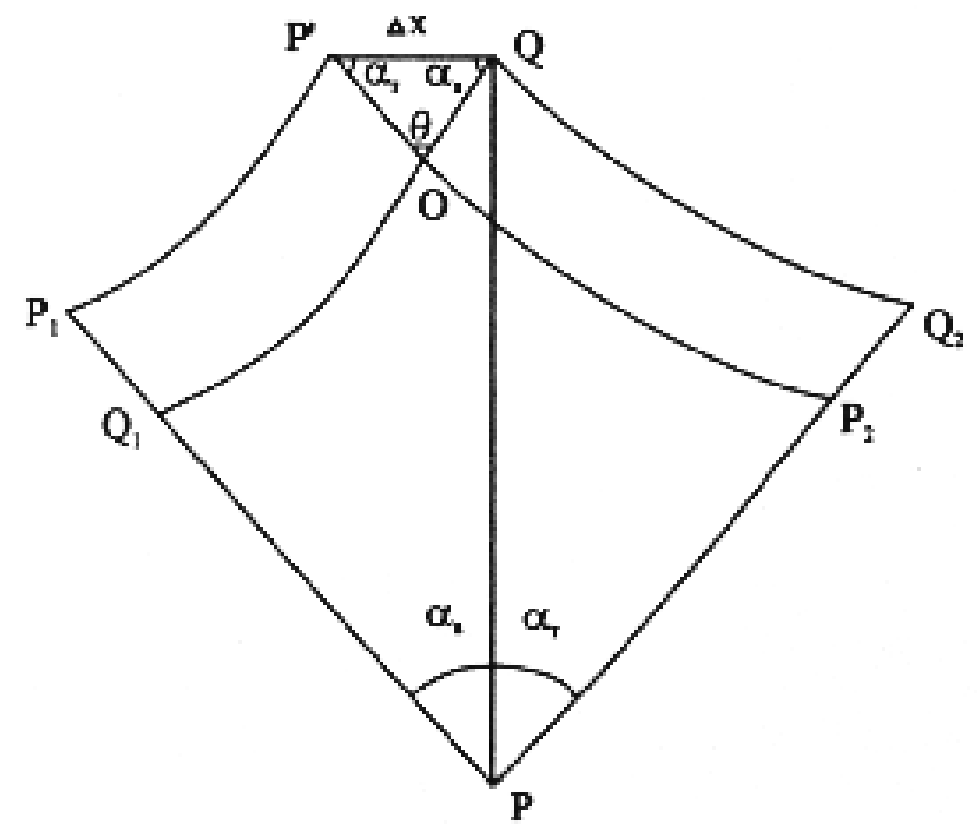

Figure 14. Migration depth-velocity relationship diagram in a general subsurface structure. (a) A wrong velocity migrates the reflection to a position $\mathrm{P}^{\prime}$ which has a lateral displacement of $\Delta x$ in addition to a vertical displacement $\Delta z$. (b) is an enlarged view of the lower part of (a).

vertical and lateral displacements from the true position $\mathrm{P}$. We denote these displacements with $\Delta x=\overline{P^{\prime} Q}$ and $\Delta z=\overline{Q P}$. In this case, the traveltime will be, 


$$
\begin{aligned}
t & =\frac{1}{\bar{v}_{m}}\left[\overline{S P^{\prime}}+\overline{P^{\prime} R}\right]=\frac{1}{\bar{v}_{m}}\left[\left(\overline{S P}-\overline{P_{1} P}\right)+\left(\overline{R P}-\overline{P_{2} P}\right)\right] \\
& =\frac{1}{\bar{v}_{m}}\left[\left(\overline{S P}-\overline{Q_{1} P}\right)+\left(\overline{R P}-\overline{Q_{2} P}\right)\right]+\frac{1}{\bar{v}_{m}}\left(\overline{P_{2} Q_{2}}-\overline{P_{1} Q_{1}}\right)
\end{aligned}
$$

From Figure 14b, the following relationship holds in the triangle $\Delta P^{\prime} Q O$,

$$
\frac{\sin \alpha_{s}}{\overline{P^{\prime} O}}=\frac{\sin \alpha_{r}}{\overline{Q O}}=\frac{\sin \theta}{\overline{\Delta x}}
$$

Thus, we have,

$$
\begin{aligned}
\overline{P_{2} Q_{2}}-\overline{P_{1} Q_{1}} & \cong \overline{Q O}-\overline{P^{\prime} O} \\
& =\frac{\Delta x}{\sin \theta} \sin \alpha_{r}-\frac{\Delta x}{\sin \theta} \sin \alpha_{s} \\
& =\frac{\Delta x}{\sin \theta}\left(\sin \alpha_{r}-\sin \alpha_{s}\right) \\
& =\frac{\Delta x}{\cos (\theta / 2)} \sin \frac{\alpha_{r}-\alpha_{s}}{2}
\end{aligned}
$$

In the derivation of the last equation above, we have used the following relationships $\alpha_{r}+\alpha_{s}+\theta=180^{\circ} ; \quad \sin \theta=2 \sin \frac{\theta}{2} \cos \frac{\theta}{2}$ and $\sin \alpha_{r}-\sin \alpha_{s}=2 \cos \frac{\alpha_{r}+\alpha_{s}}{2} \sin \frac{\alpha_{r}-\alpha_{s}}{2}$. Therefore, the distance part of the last term of (16) would be an order smaller than $\Delta x$ as long as $\left|\sin \left(\alpha_{r}-\alpha_{s}\right) / 2\right|<0.10$ and $\theta$ is not close to $180^{\circ}$. The latter condition generally holds, as $\alpha_{s}$ and $\alpha_{r}$ would not be zero for most cases. The first condition is equivalent to $\left|\alpha_{s}-\alpha_{r}\right|<12^{\circ}$, i.e., the difference between the two illuminating angles being less than $12^{\circ}$. As $\left|\alpha_{s}-\alpha_{r}\right|=2 \alpha$ where $\alpha$ is the structural dip at $\mathrm{P}$, the above inequality thus only holds for structures of gentle dip. In such cases, (16) can be properly approximated by

$$
t=\frac{1}{\bar{v}_{m}}\left[\left(\overline{S P}+\overline{Q_{1} P}\right)+\left(\overline{R P}-\overline{Q_{2} P}\right)\right]
$$

This equation is physically equivalent to the assumption that the lateral displacement $\Delta x$ is negligible compared to the vertical one. Eliminating $t$ from equations (15) and (19), we obtain,

$$
(1-\beta)(\overline{S P}+\overline{R P})=\overline{Q_{1} P}+\overline{Q_{2} P}
$$


where $\beta=\frac{\bar{v}_{m}}{\bar{v}}$. From Figure 14, the following general relations hold,

$$
\begin{aligned}
& \overline{S P}=z / \cos \alpha_{s} ; \quad \overline{Q_{1} P}=-\Delta z \cos \alpha_{s} ; \\
& \overline{R P}=z / \cos \alpha_{r} ; \quad \overline{Q_{2} P}=-\Delta \cos \alpha_{r}
\end{aligned}
$$

Substituting these relations into (20) leads to,

$$
\Delta z=(\beta-1) \frac{z}{\cos \alpha_{s} \cos \alpha_{r}}
$$

In the case of zero offset source-receiver pair just at D, $\alpha_{s}=\alpha_{r}=0$, we obtain,

$$
\Delta z=(\beta-1) z
$$

This implies that the migration depth $z$ will be shallower than the true depth $\mathrm{z}$ if $\mathrm{a}$ smaller than the true velocity $\left(\bar{v}_{m}<\bar{v}\right)$ is used for migration, while deeper if a higher velocity $\left(\bar{v}_{m}>\bar{v}\right)$ is used. Only when $\beta=1$, i.e., the true medium velocity is used for migration, will the diffractor be properly located. By denoting $\Delta z_{0}=(\beta-1) z,(21)$ can be rewritten as,

$$
\Delta z\left(\alpha_{s}, \alpha_{r}\right)=\frac{\Delta z_{0}}{\cos \alpha_{s} \cos \alpha_{r}}
$$

Now let's consider the following three categories.

\section{Migration velocity less than the true velocity $\left(\bar{v}_{m}<\bar{v}\right)$}

In this case, $\beta<1, \Delta z_{0}<0$, and $\Delta z\left(\alpha_{s}, \alpha_{r}\right)<\Delta z_{0}$. Generally the following relation also holds,

$$
\Delta z\left(\alpha_{s}+\varepsilon_{1}, \alpha_{r}+\varepsilon_{2}\right)<\Delta z\left(\alpha_{s}, \alpha_{r}\right)
$$

for any $\alpha_{s}, \alpha_{r}$ and any non-negative values of $\varepsilon_{1}, \varepsilon_{2}$. This relation indicates that the migration image of $\mathrm{P}$ will form a "smile" which curves upward on a common image gather (CIG) which is a display of migration traces versus the source-receiver offset corresponding to a fixed surface point.

\section{Migration velocity greater than the true velocity $\left(\bar{v}_{m}>\bar{v}\right)$}

In this case, $\beta>1, \Delta z_{0}>0$, and $\Delta z\left(\alpha_{s}, \alpha_{r}\right)>\Delta z_{0}$. Similar to (24) we have,

$$
\Delta z\left(\alpha_{s}+\varepsilon_{1}, \alpha_{r}+\varepsilon_{2}\right)>\Delta z\left(\alpha_{s}, \alpha_{r}\right)
$$


This relation indicates that the migration image of $\mathrm{P}$ forms a "frown" which curves downward on a CIG.

\section{Migration velocity equal to the true velocity $\left(\bar{v}_{m}=\bar{v}\right)$}

In this special case, $\beta=1, \Delta z_{0}=0$, thus,

$$
\Delta z\left(\alpha_{s}, \alpha_{r}\right)=0
$$

for any source-receiver pair. This simply means that when the true velocity is used for migration $\left(\bar{v}_{m}=\bar{v}\right)$, the migration images of the diffractor point $\mathrm{P}$ will be at the exact depth regardless of source-receiver offset. So, its images form a horizontal segment on the CIG displays.

To consider prestack migration velocity analysis in terms of offset and common midpoints, consider again Figure 8 for a point diffractor at $(0, z)$. The midpoint can be denoted by $X=\left(x_{r}+x_{s}\right) / 2$ and the offset denoted by $2 h$ so that $x_{s}=X-h$ and $x_{r}=X+h$. Equation (10) gives the total traveltime for a particular point diffractor, but it can be embedded into an equation for a "correct" migration ellipse,

$$
t_{1}=t_{1}(X)=\frac{1}{v}\left(\sqrt{(X-h-x)^{2}+z^{2}}+\sqrt{(X+h-x)^{2}+z^{2}}\right)
$$

In terms of migration velocity and migration coordinates, we can also write the traveltime expression as,

$$
t_{2}=t_{2}(X)=\frac{1}{v_{m}}\left(\sqrt{\left(X-h-x_{m}\right)^{2}+z_{m}^{2}}+\sqrt{\left(X+h-x_{m}\right)^{2}+z_{m}^{2}}\right)
$$

When $\bar{v}_{m}=\bar{v}$, the migration ellipse (28) is identical to the ellipse (27) for the true velocity; so if the velocity analysis location $x_{m}$ coincides with the diffractor location $x=0$, we then have $z_{m}=z$ for all $h$. That is, the migration depth equals the correct depth of the diffractor regardless of the offset value when the velocity is correct. When the velocity is not correct $\left(v_{m} \neq v\right)$, we need to evaluate the envelope of migration ellipses for all values of the midpoints $X$. That is, we need to set the derivatives of $t_{1}(X)$ equal to the derivatives of $t_{2}(X)$ for all $X$. If, again, we perform the velocity analysis at the actual diffractor location, then these derivatives give, respectively, the slopes of the correct and the incorrect migration ellipses at $x_{m}=x=0$. These slopes are different unless both ellipses are flat at the analysis location, i.e., unless $X=0$ also. So we set $X=x_{m}=x=0$ into the expressions for $t_{1}(X)$ and $t_{2}(X)$ to obtain,

$$
\frac{h^{2}+z^{2}}{v^{2}}=\frac{h^{2}+z_{m}^{2}}{v_{m}^{2}}
$$


or

$$
z_{m}^{2}=\left(\beta^{2}-1\right) x^{2}+\beta^{2} z^{2}
$$

where $\beta=\frac{v_{m}}{v}$.

In the case of $v_{m}<v$, we have $\beta<1$. And (30) can be rewritten as,

$$
z_{m}^{2}+\left(1-\beta^{2}\right) x^{2}=\beta^{2} z^{2}
$$

which is just an ellipse. So this is just a special case of (24).

If $v_{m}>v$, then $\beta>1$, and (30) is essentially the following hyperbolic equation,

$$
z_{m}^{2}-\left(\beta^{2}-1\right) x^{2}=\beta^{2} z^{2}
$$

This is an hyperbolic smile in the half plane of positive depth.

If $v_{m}=v$, then $\beta=1$, and (30) simplifies to $z_{m}=z$. This represents a horizontal line segment. Only in this special case of using the correct velocity for migration, will the final stack of the migrated CRP gather reach its highest energy in the migration section.

The above procedure of migrating a CRP gather can also be geometrically represented. Figure 15 shows a CRP gather from the same diffractor model of Figure 8. This gather is essentially equivalent to that of CMP gather in this special case of a single diffractor point in constant velocity medium. Figure 16 shows the migrated CRP gathers for this diffractor model corresponding to different migration velocities. As the migrated CRP gather is just the same as the so-called CIG gathers, Figure 16 thus equivalently illustrates the CIGs corresponding to different migration velocities. It shows that the recorded hyperbola in the CRP gather will appear to be elliptical smiles when a

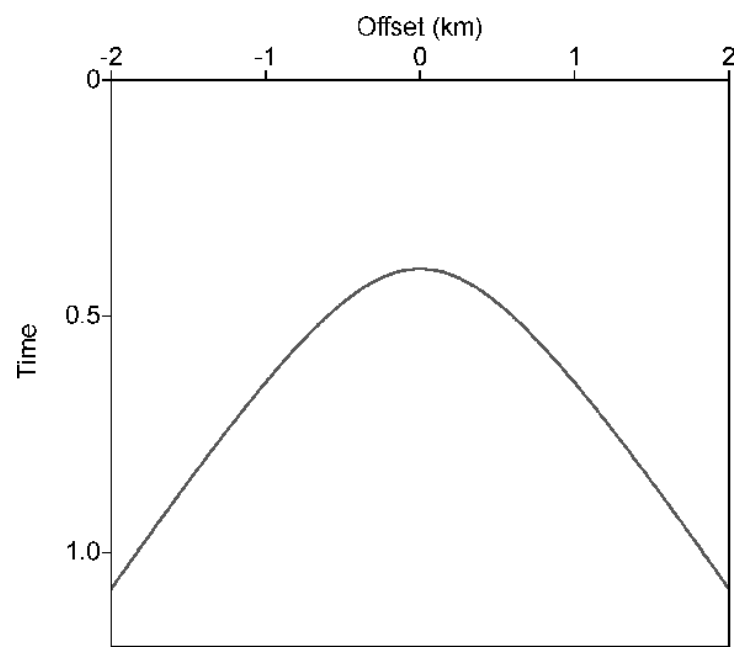

Figure 15. Common reflection point (CRP) gather from a diffractor point model. The diffractor lies at $0.8 \mathrm{~km}$ depth in the middle of the model. smaller velocity is used for migration, while a hyperbolic frown when a larger velocity is used for migration. If the correct velocity is used, then the CIG gather will show a horizontally aligned segment. 

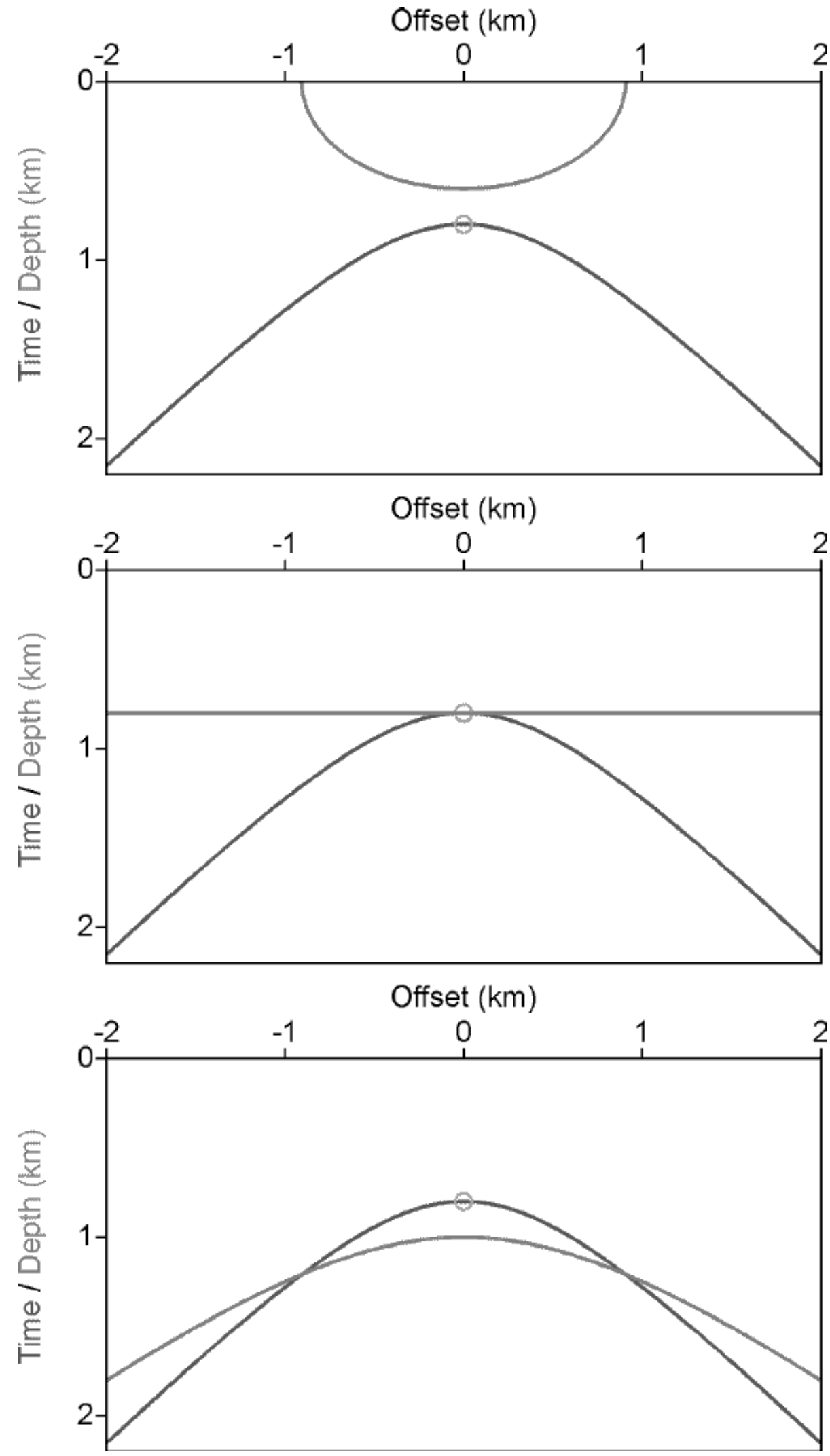

Figure 16. Common image gathers when different migration velocities are used. Migration velocity is $3 \mathrm{~km} / \mathrm{s}$ (top), $4 \mathrm{~km} / \mathrm{s}$ (middle) and $5 \mathrm{~km} / \mathrm{s}$ (bottom) respectively. The circle in light grey represents the diffractor position. The dary grey curve is the scaled CRP gather. 

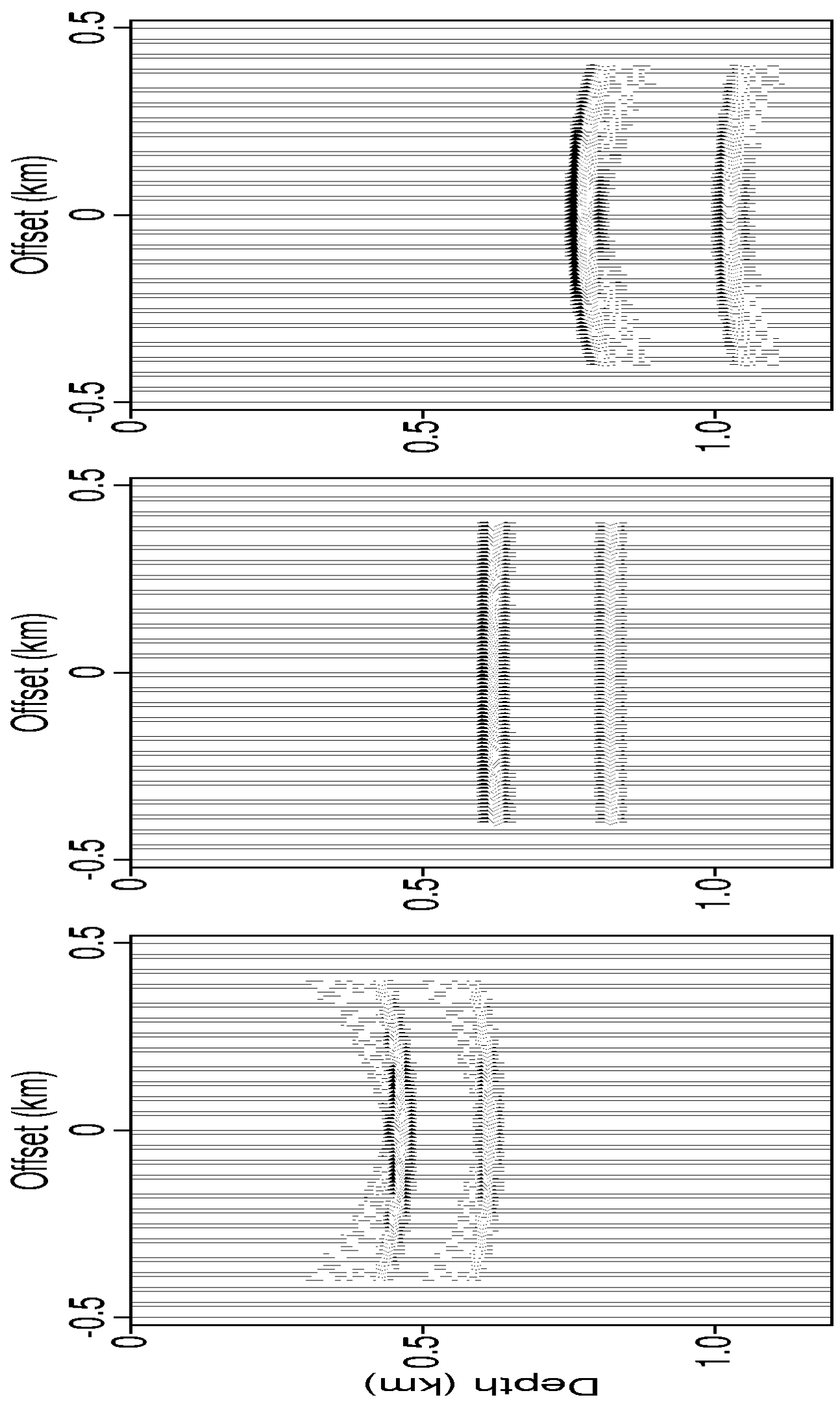

Figure 17. Common image gathers for a two point diffractors model. 
Therefore, the velocity error in migration is very well expressed on common image gathers. We can use the same point diffractors model to illustrate the above theoretical observations. 81 shot profiles are theoretically simulated, with each record consisting of 60 traces. Figure 17 shows the CIGs for surface position $x=1.0 \mathrm{~km}$ when different velocities are used in migration. It clearly demonstrates that only when the true velocity is used, will the migration images of diffractors be independent of the source-receiver offset. When the velocity is lower than the true velocity $\left(v_{m}=\right.$ $3,000 \mathrm{~m} / \mathrm{s}$ ), the diffractor images form "smiles" at a depth shallower than the true depth. This is totally in agreement with equations (24) and (31). In contrast, when the

velocity is higher than the true velocity $\left({ }^{v_{m}}=5,000 \mathrm{~m} / \mathrm{s}\right)$, the diffractors will be expressed as frowns on a CIG at a depth greater than the true depth. This is just what has been predicted by the mathematical expressions (25) and (32).

Thus, the migration velocity error is well documented on both the the final migration sections (Figure 13) and the CIGs (Figure 17). Interestingly enough, the CIG gathers show shallow smiles for low velocities and deep frowns for high velocities (Lines et al., 1993), whereas the final depth migration sections show shallow frowns for low velocities and deep smiles for high velocities (Yilmaz, 1987).

If we further take a careful look at Figure 13 and Figure 17, it is seen that the curvatures of the shallow "smiles/frowns" are generally larger than the deep ones. This indicates that velocity errors are more pronounced on shallow reflections and thus are easier to be corrected. This is also in agreement with the general observation that sufficient offset/depth ratio should be kept in order to properly analyze the velocity errors (Lines, 1993). Luckily we often have more constraints available on the shallow parts of the earth, such as well logging and geological exposures. We can also more effectively use techniques such as first break tomography to constrain our near surface velocity estimation. With respect to the deeper structure, we generally have to accept that it is coarsely defined and more ambiguous.

Though the diffractor model is over simplified, it is of vital significance in migration and velocity analysis theory, as any complicated structures can be considered as a continuum of diffractors. This is especially suitable for moveout analysis on a CIG which corresponds to a single surface point. These "smiles" and "frowns" patterns on CIGs can be effectively used to qualitatively and quantitatively analyze the migration velocity (Al-Yahya, 1987).

\section{CONCLUSIONS}

Depth migration is very sensitive to errors in the velocity model. Migration moveout features such as "smiles" and "frowns" have previously been reported (ref. Yilmaz, 1987; Lines et al., 1993). We have shown both mathematically and geometrically these "smiles" and "frowns" on migration sections and CIGs. Using the simple diffractors model, we demonstrated that after migration, either using the stacked data or the prestack shot gathers, the diffractions will be migrated to shallow "frowns" when the migration velocity is too small, and deep "smiles" when the velocity is too large. Only when the migration velocity is exactly the medium velocity, will both the poststack and prestack migration produce concentrated "blobs" 
in the migration section. However, it is the common image gathers produced in the prestack depth migration that provide an effective migration velocity analysis domain. Our study starting from the very general subsurface structure showed that the migration moveout in the CIGs are interestingly different from the patterns in the migration sections. In CIGs, a lower velocity produces shallow smiles while a higher velocity results in deeper frowns. When the migration is correct, the CIGs presents a horizontal segments at the exact diffractor depths which indicate that the migration image of the point diffractor is independent of source-receiver offset. These moveout patterns of "smiles"and "frowns" can serve as both qualitative and quantitative criteria for migration velocity analysis.

\section{ACKNOWLEDGMENTS}

We would like to thank Jianhua Pan for his technical assistance. The authors also thank Sven Treitel for providing us a copy of the Maeland paper. Most of the funding and research for this project was provided by Memorial University Seismic Imaging Consortium and we are grateful for this support.

\section{REFERENCES}

Al-Yahya, K., 1989, Velocity analysis by iterative profile migration: Geophysics, 54, 718-729.

Claerbout, J. F.,1985, Imaging the earth’s interior: Blackwell Scientific Publ..

Lines, L. R., 1993, Ambiguity in analysis of velocity and depth (short note): Geophysics, 58, 596-597.

Lines, L. R., Rahimian, F. and Kelly, K. R., 1993, A model-based comparison of modern velocity analysis methods: The Leading Edge, 12, 750-754.

Loewenthal, D., Lu, L., Roberson, R. and Sherwood, J., 1976, The wave equation applied to migration: Geophys. Prosp., 24, 380-399.

Maeland, E., 1989, Focusing aspects of zero-offset migration: Geophysical Transactions, 35, No.3, $145-156$.

Robinson, E. A., and Treitel, S., 1980, Geophysical signal analysis: Prentice-Hall Book Co.

Scales, J. A., 1995, Theory of seismic imaging: Springer-Verlag.

Sheriff, Robert E., 1991, Encyclopedic dictionary of exploration geophysics: Soc. Expl. Geophys.

Sneddon, I. N., 1957, Elements of partial differential equations: McGraw-Hill Book Co.

Whitmore, N. D. and Garing, J. D., 1993, Interval velocity estimation using iterative prestack depth migration in the constant angle domain: The Leading Edge, 12, 757-762.

Yilmaz, Ö, 1987, Seismic data processing: Society of Exploration Geophysicists. 